\title{
Pathologic Response During Chemo-radiotherapy and Variation of Serum VEGF Levels Could Predict Effects of Chemo-Radiotherapy in Patients with Esophageal Cancer
}

\author{
Jing-Ping Yu, Wen-Bin Lu, Jian-Lin Wang, Xin-Chu Ni, Jian Wang, Zhi-Qiang \\ Sun, Su-Ping Sun*
}

\begin{abstract}
Background: To investigate the relationship between pathologic tumor response to concurrent chemoradiotherapy and variation of serum VEGF in patients with esophageal cancer. Materials and Methods: Forty six patients with esophageal cancer who were treated with concurrent chemo-radiotherapy were enrolled. Endoscopic and pathologic examination was conducted before and four weeks afterwards. Serum level of VEGF was documented before, four weeks later and after chemo-radiotherapy. The relationship between pathologic response and the variation of serum level of VEGF and its influence on the prognosis were investigated. Results: Serum level of VEGF decreased remarkably during and after chemo-radiotherapy in patients whose pathologic response was severe $(F=5.393,4.587, P(0.05)$. There were no statistical differences of serum VEGF level before, during and after chemo-radiotherapy for patients whose pathologic response was moderate or mild. There were $18(85.7 \%), 7(53.8 \%)$ and 6 patients $(50.0 \%)$ whose serum VEGF level dropped in the severe, moderate and mild group, respectively, with significant differences among these groups $(\mathrm{p}=\mathbf{0 . 0 4 6})$. Two year survival rates of patients with severe, moderate and mild pathologic response were $61.9 \%, 53.8 \%$ and $33.3 \%$ respectively, and no statistically difference between severe and mild group regarding $O S(p=0.245)$ was tested. Conclusions: Tumor pathologic response during chemo-radiotherapy and the changes of serum VEGF lever could predict curative effects of chemo-radiotherapy in patients with esophageal cancer.
\end{abstract}

Keywords: Esophageal carcinoma - pathology response - vascular endothelial growth factor - prognosis

Asian Pac J Cancer Prev, 16 (3), 1111-1116

\section{Introduction}

Most patients with esophageal cancer are unable or refuse to receive surgical treatment due to advanced disease or with other medical reasons, and concurrent chemotherapy could serve as an option for this cohort of patients. Previous study suggested that serious pathological reaction of tumor tissue after radiotherapy could be associated with higher long-term survival rate (Brun et al., 2001). Some recent studies have found that the expression of vascular endothelial growth factor (VEGF) in the tissue of patients with esophageal cancer is associated with blood vessel invasion as well as lymphatic metastasis and survival, and acts as an independent prognostic factor of esophageal cancer (Jiang et al., 2012; Angelescu et al., 2013; Peng et al., 2013; Cellini et al., 2014; Wang et al., 2014). However, studies on serum VEGF levels and the radiosensitivity of patients with esophageal cancer are rarely reported. This study hypothesizes that there is relationship between pathological reaction of tumor tissue and the changes in serum levels of VEGF in patients with esophageal cancer during concurrent radiotherapy, and there is relationship between these two factors and the prognosis.

\section{Materials and Methods}

\section{Clinical data}

46 patients with esophageal cancer treated for the first time (with age $\leq 75$, KPS score $\geq 80$ ) and hospitalized in the department of radiotherapy of Changzhou No.2 Hospital in Jiansu Province from January, 1, 2008 to December, 31,2010 are enrolled in this study. All of the cases have been diagnosed with esophageal squamous carcinoma through a biopsy with an esophagoscope before the treatment and identified by disease stages of stage I to IV (according to the staging criteria of non-surgical treatment of esophageal cancer in 2010), and have been unable or refused to receive the surgery due to medical reasons. See the general information of all the patients in Table.1. 


\section{Radiotherapy equipment and apparatus}

German Siemens Primus-H medical linear accelerator, Siemens 128-layer dual-source CT simulation positioning machine; Nucletron-HP digital simulation positioning machine; American CMS treatment planing system; German Siemens Lantis network system, etc.

\section{Design of outlining and planning of radiotherapy target region}

The three-dimensional conformal technology and CT-SIM design radiotherapy plan were adopted; therein, the gross tumor volume (GTV) included primary lesions and lymph metastases; the clinical target volume (CTV) included subclinical lesions and potentially involved lymph node regions. The planning target volume (PTV) expanded by $1 \mathrm{~cm}$ on all sides based on the CTV, and by 3 to $5 \mathrm{~cm}$ from top to bottom. The number and the design of the radiation fields depended upon the specific circumstance of the target region.

\section{Chemoradiotherapy scheme}

The pattern of conventional dose fractionation was adopted to ensure GTV with a radiation dose of 60 to 64 Gy (30 to 32 times, 6 to 7 weeks), CTV with a radiation dose of $50 \mathrm{~Gy}$ ( 25 times, 5 weeks) and spinal cord with a radiation dose no more than 40 to 45 Gy (4 to 6 weeks). Excessive radiation doses on the lung and the heart were avoided as much as possible, and the esophageal cancer in cervical segments and the upper thoracic portion received a double supraclavicular prophylactic irradiation. All of the patients were treated by $6 \mathrm{MV} X$ ray, with a fractionated dose of 1.8 to $2.0 \mathrm{~Gy}$, five times a week. The radiotherapy scheme consisted of Paclitaxel Liposome Injection 135 $\mathrm{mg} / \mathrm{m} 2$, for the first day; Cisplatin (DDP) $20 \mathrm{mg} / \mathrm{m}^{2}$ for the second to fifth days, and an intravenous drip on day 21 as a period.

Classification criteria of the pathological reaction of postradiotherapy tumor tissues

The gastroscopy was conducted when the radiation dose reached $40 \mathrm{~Gy}$. The radiation reaction was classified into three grades according to the pathological reaction of the postradiotherapy tumor tissue[2]: severe reaction, moderate reaction and mild reaction.

\section{Measurement method for serum VEGF level}

The blood of all the patients was sampled 1 week before chemoradiotherapy, 4 weeks after chemoradiotherapy and within 1 week after chemoradiotherapy. Two ml peripheral venous blood was extracted, blended and centrifuged at 4 for $10 \mathrm{~min}$ at $3,000 \mathrm{rpm} / \mathrm{min}$ by a centrifugal radium of 10 $\mathrm{cm}$, and the isolated serum was saved in a refrigerator at $-700 \mathrm{C}$ for subsequent examination. A group of 30 healthy examinees constituted a healthy control group. A doubleantibody sandwich ABC-ELISA method was used and performed according to the standard operating protocol. The VEGF kit was manufactured by China International Capital Corporation Limited.

\section{Evaluation of the short-term curative effect}

The esophagus X-ray barium radiography was conducted before chemoradiotherapy, 4 weeks after chemoradiotherapy and 1 week after chemoradiotherapy. The short-term curative effect was evaluated based on the curative effect criteria of WHO and classified into 4 grades[2]: complete remission (CR), partial remission (PR), stable disease (SD) and progression disease (PD). $\mathrm{CR}+\mathrm{PR}$ was regarded as effective, and $\mathrm{SD}+\mathrm{PD}$ as ineffective.

\section{Follow-up visit and statistical analysis}

The follow-up visits were terminated on December, 31 , 2012. All of the patients were followed up for 2 years with three patients missing the follow-up. The follow-up rate of the patients was $93.5 \%$. SPSS 19.0 software package was employed for statistical analysis. The measurement data are all expressed in the form of ; the comparison of the rates among multi-group count data was performed by using 2 test; the comparison of the means among multi-group measurement data was performed by using analysis of variance; the survival rate was calculated by the method of Kaplan-Meier; Log-rank method was adopted to determine a statistical significance. Two-sided test level of $\mathrm{p}<0.05$ was considered to be statistically significant.

\section{Results}

Influence of chemoradiotherapy on the histological reaction of esophageal cancer

Gastroscopic biopsies on the 46 patients with esophageal cancer in the fourth week of the treatment indicated that: 21 cases revealed severe reaction in tumor tissue $(45.7 \%), 13$ cases revealed moderate reaction (28.2\%), and 12 cases revealed a mild reaction (26.1\%). There was no statistically significant difference in the pathological reaction between the tumor tissues of patients of different genders, ages, tumor sites, tumor types, $\mathrm{T}$ stages, $\mathrm{N}$ stages, $\mathrm{M}$ stages and TNM stages during chemoradiotheray.

\section{Influence of chemoradiotherapy on serum VEGF level of patients with esophageal cancer}

The mean levels of serum VEGF of 46 patients with esophageal cancer before, during and after the treatment were $(116.5 \pm 39.1),(106.5 \pm 25.4)$ and $(104.0 \pm 23.0) \mathrm{ng} / \mathrm{L}$ respectively, obviously higher than the mean level of serum VEGF ((79.6 \pm 39.2$) \mathrm{ng} / \mathrm{L})$ of the healthy control group, and the difference was statistically significant $(\mathrm{F}=8.329, \mathrm{p}=0.000)$. The serum VEGF levels of cases after treatment were significantly lower than their levels before treatment $(\mathrm{F}=4.018 ; \mathrm{p}=0.047)$. However, the serum VEGF levels of 31 cases $(67.4 \%)$ after the treatment declined as compared to those before the treatment, and those of another 15 cases $(32.6 \%)$ increased. The differences of the change in the serum VEGF level of patients of different genders, ages, tumor sites, tumor types, $\mathrm{T}$ stages, $\mathrm{N}$ stages, $\mathrm{M}$ stages and TNM stages before, during and after the treatment were not statistically significant.

The correlation between the pathological reaction of the esophagus tissue and the expression level of serum VEGF during treatment

As shown in Table.2, the difference between the 
Pathologic Response and Variation of Serum VEGF Could Predict Chemo-Radiotherapy Response of Esophageal Cancer

mean levels of serum VEGF of patients with esophageal cancer revealing severe reaction and the group revealing mild reaction was statistical significance $(\mathrm{F}=6.800$, $\mathrm{p}=0.016$ ); while, the differences of the mean levels of serum VEGF between the severe reaction group and the moderate reaction group as well as between the moderate reaction group and the mild reaction group before the treatment were all statistically insignificant $(\mathrm{F}=1.509,0.926, \mathrm{p}>0.05)$. The serum VEGF levels of the group revealing severe reaction after chemoradiotherapy presented a gradual decline; the decreases of serum VEGF level before the treatment compared with those during and after the treatment were both statistically significant $(\mathrm{F}=5.393,4.587, \mathrm{p}<0.05)$. However, the difference between serum VEGF levels of those after the treatment and during the treatment was not statistically significant $(\mathrm{F}=0.008, \mathrm{p}>0.05)$. The serum VEGF levels of the group revealing moderate reaction presented a decreasing and then increasing trend, and those of the group revealing mild reaction presented an increasing and then decreasing trend; but there was no statistical significant difference between the serum VEGF levels of any two circumstances (before, during and after the treatment) $(\mathrm{F}=0.253,2.111$, $\mathrm{p}>0.05)$. Therefore, the numbers of cases showing a decline of serum VEGF level in the group revealing severe reaction, the group revealing moderate reaction and the group revealing mild reaction after the treatment were respectively $18(85.7 \%), 7(53.8 \%)$ and $6(50.0 \%)$ in comparison with their serum VEGF levels before the treatment. The differences among the three groups were statistically significant. $(2=6.072, \mathrm{p}<0.05)$.

The relationship between the pathological reaction \& serum VEGF change during chemoradiotherapy and the long-term survival of patients with esophagus cancer

Table 1. General Information of All 46 Patients with Esophagus Cancer

\begin{tabular}{|c|c|c|c|}
\hline \multicolumn{2}{|l|}{ Item } & N (Person) & Proportion \\
\hline \multicolumn{4}{|l|}{ Gender } \\
\hline Male & & 33 & $71.7 \%$ \\
\hline Female & & 13 & $28.3 \%$ \\
\hline \multicolumn{2}{|l|}{ Median age } & $66(40 \sim 75)$ & -- \\
\hline \multicolumn{4}{|l|}{ Tumor site } \\
\hline \multicolumn{2}{|l|}{ The upper thoracic portion } & 11 & $23.9 \%$ \\
\hline \multicolumn{2}{|l|}{ The mid-thoracic portion } & 21 & $45.7 \%$ \\
\hline \multicolumn{2}{|l|}{ The lower thoracic portion } & 14 & $30.4 \%$ \\
\hline \multicolumn{4}{|l|}{ Tumor classification } \\
\hline \multicolumn{2}{|l|}{ Medullary type } & 43 & $93.5 \%$ \\
\hline \multirow{5}{*}{$\begin{array}{l}\text { Fungating type+ulcerative type } \\
\text { T stages }\end{array}$} & 3 & $6.5 \%$ & \\
\hline & $\mathrm{T} 1$ & 4 & $8.7 \%$ \\
\hline & $\mathrm{T} 2$ & 26 & $56.5 \%$ \\
\hline & $\mathrm{T} 3$ & 15 & $32.6 \%$ \\
\hline & $\mathrm{T} 4$ & 1 & $2.2 \%$ \\
\hline \multirow[t]{3}{*}{$\mathrm{N}$ stages } & NO & 15 & $32.6 \%$ \\
\hline & N1 & 26 & $565 \%$ \\
\hline & $\mathrm{N} 2$ & 5 & $10.9 \%$ \\
\hline \multirow[t]{2}{*}{ M stages } & NO & 39 & $84.8 \%$ \\
\hline & N1 & 7 & $15.2 \%$ \\
\hline \multirow[t]{4}{*}{ TNM stages } & I & 13 & $283 \%$ \\
\hline & II & 23 & $50 \%$ \\
\hline & III & 3 & $6.5 \%$ \\
\hline & IV & 7 & $152 \%$ \\
\hline
\end{tabular}

There were 27 death cases $(58.7 \%)$ among the 46 patients; wherein, 1 case $(3.7 \%)$ died of radiation pneumonia; 1 case (3.7) died of the secondary neoplasia (kidney cancer); 3 cases $(11.1 \%)$ died from the failure of local tumor control; 6 cases $(22.2 \%)$ died of tumor metastasis (lung, abdominal cavity, bone and widespread metastasis); 16 cases (59.2\%) died of tumor relapse. A total of 19 patients (41.3) still survived, among which, the numbers of surviving patients in the group were due to a severe reaction, moderate reaction and mild reaction were respectively 10 (47.6\%), 6 (46.2) and 3 (25.0\%). There was no statistical difference among the three groups $(2=2.477, p=0.286)$. See details in Table.3.

The two-year survival rates of patients in the group revealing severe reaction, the group revealing moderate reaction and the group revealing mild reaction were respectively $61.9 \%, 53.8 \%$ and $33.3 \%$. The median survival time of the group revealing severe reaction extended longer than that of the group revealing mild reaction (29.8 months, 95\% CI: 16.9 to $42.7 \mathrm{VS} 18.7$ months, $95 \%$ CI: 1.9 to $35.5 \%)$ ). However, the comparison of overall survivals (OS) of the groups revealing severe reaction and mild reaction showed no statistical significance $(2=1.351, \mathrm{p}=0.245)$, as described in Figure 1 .

The relationship between the serum VEGF change and the short-term curative effects \& prognosis of the patients with esophagus cancer after chemoradiotherapy

There were 31 patients in total enrolled in the group $(67.4 \%)$ whose serum VEGF levels declined after treatment compared with those before treatment. Additionally, the serum VEGF levels of another 15 patients $(32.6 \%)$ increased. Based on the short-term curative effect evaluation, there are 22 patients $(71.0 \%)$ with declining serum VEGF level and 6 patients $(40.0 \%)$

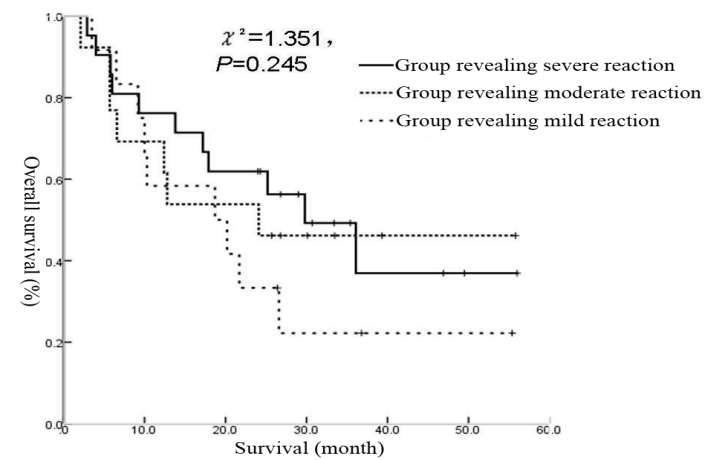

Figure 1. The Overall Survival Rate of the Groups with Different Pathological reactions

Table 2. Changes in Serum VEGF Levels of Pathological Reaction Groups During Different Chemoraiotherapy Periods of Esophagus Cancer

\begin{tabular}{lcccc}
\hline $\begin{array}{c}\text { Group Number of } \\
\text { patients }\end{array}$ & $\begin{array}{c}\text { Before } \\
\text { treatment }\end{array}$ & $\begin{array}{c}\text { During } \\
\text { treatment }\end{array}$ & $\begin{array}{c}\text { After } \\
\text { treatment }\end{array}$ \\
\hline Severe & 21 & $129.3 \pm 46.6^{\mathrm{a}, \mathrm{b}, \mathrm{c}}$ & $104.1 \pm 16.9^{\mathrm{b}}$ & $104.7 \pm 24.3^{\mathrm{c}}$ \\
Moderate & 13 & $111.0 \pm 33.5$ & $102.0 \pm 33.7$ & $106.0 \pm 30.0$ \\
Mild & 12 & $100.2 \pm 20.8 \mathrm{a}$ & $115.5 \pm 27.5$ & $100.5 \pm 10.9$ \\
\hline
\end{tabular}

Comparison of serum VEGF level between the group revealing severe reaction and the group revealing mild reaction before the treatment, $\mathrm{F}=6.800, \mathrm{p}=0.016$ 
Table 3. The Relationship between the Reaction Degree of Tumor Tissue and the Long-term Curative Effects \& Prognosis of 46 Patients

\begin{tabular}{lccccc}
\hline Group & Number of patients & \multicolumn{2}{c}{ Short-term curative effect } & \multicolumn{2}{c}{ Number of existing survivors } \\
& & Effective number & Ratio(\%) & Occurrence number & Ratio (\%) \\
\hline Severe & 21 & 19 & 90.5 & 10 & 47.6 \\
Moderate & 13 & 5 & 38.5 & 6 & 46.2 \\
Mild & 12 & 4 & 33.3 & 3 & 25 \\
2 & & & 14.289 & & 2.477 \\
P value & & & $<0.05$ & & $>0.05$ \\
\hline
\end{tabular}

Table 4. The Relationship of the Serum VEGF Changes and the Short-term Curative Effects AND Prognosis of 46 Patients

\begin{tabular}{|c|c|c|c|c|c|}
\hline \multirow[t]{2}{*}{ VEGF change } & \multirow{2}{*}{$\begin{array}{c}\text { Number } \\
\text { of patients }\end{array}$} & \multicolumn{2}{|c|}{ Short-term curative effect } & \multicolumn{2}{|c|}{ Number of existing survivors } \\
\hline & & Effective number & Ratio $(5 \%)$ & Occurrence number & Ratio (\%) \\
\hline Declining group & 31 & 22 & 71 & 18 & 58.1 \\
\hline Rising group & 15 & 6 & 40 & 6 & 40 \\
\hline 2 & & & 4.07 & & 0.697 \\
\hline$P$ value & & & $<0.05$ & & $>0.05$ \\
\hline
\end{tabular}

with rising serum VEGF level who were determined as the effective ones. There was a statistical significance in the difference between two groups $(2=4.070, \mathrm{p}<0.05)$. The numbers of patients of the two groups with an overall survival of more than 2 years were respectively $18(58.1 \%)$ and $6(40.0 \%)$. There was no statistical significance in the difference between the two groups, as embodied in Table.4.

\section{Discussion}

The main method of treatment for cancer is multidisciplinary (Tian et al., 2014; Liu et al., 2014; Qian et al., 2014; Xiao et al., 2014). However, more than $60 \%$ of the patients cannot receive such surgery for medical reasons like local invasion and metastasis. Previous studies have suggested that five-year survival rate of concurrent chemoradiotherapy for esophageal cancer is approximate to that of surgical treatment (Shibata et al., 2002). Therefore, the US National Comprehensive Cancer Network and the Japanese Guidelines for Esophagus Cancer Treatment have also successively taken the nonsurgical treatment as the standard treatment scheme. Currently, the key issue that requires special attention is an evaluation of the curative effect of concurrent chemoradiotherapy on a real-time basis and adjusts correspondingly the treatment scheme in accord with the evaluation results.

In this study, we analyzed the relationship between the pathological reactions and clinicopathologic features of esophageal cancer after preoperative radiotherapy, and found that there were no significant differences among patients with different degrees of pathological reactions in terms of gender, age or length distribution of diseased regions, and that the clinicopathologic features were independent of the degrees of pathological reactions after chemoradiotherapy.

Studies have shown that the growth and spread of the tumor is dependent upon angiogenesis, and VEGF is currently among the most potent and specific angiogenic factors which plays an important role in the relapse and metastasis of esophageal cancer. Thus, VEGF serves as an independent prognostic factor of esophageal cancer (Crehange et al., 2007; Stahl et al., 2009). It was found by the research group in their preliminary study that the serum VEGF level was closely related with the load of tumor (Srivastava et al., 2014). The higher VEGF levels in circulating blood was presumably produced by esophageal tumor tissues. As the treatment progressed, the size of the tumor decreased, the secreted VEGF was reduced and the corresponding serum VEGF level declined at the same time. In this study, the serum VEGF levels of patients with esophageal cancer before, during and after the treatment were all elevated in comparison with those of healthy controls, and the difference between the two groups showed a statistical significance. The VEGF levels of cases after treatment were lower than their levels before treatment and the difference was statistically significant. As for the group revealing severe reaction, the serum VEGF level was high before chemoradiotherapy, and with the progressing of radiotherapy, the serum VEGF level gradually lowered; however, in the group revealing mild reaction, the serum VEGF level was low before radiotherapy, and presented an initial rising and then declining change as the chemoradiotherapy was carried forward. It was implied that the curative effect of the group with a rising serum VEGF level after chemoradiotherapy was poor. The patients with a good curative effect presented a rapidly and remarkably declining serum VEGF level during treatment, while the patients with a rising serum VEGF level possibly resisted or were non-sensitive to the radiotherapy. The result also implied that the serum VEGF level before treatment may possibly become a predicative index for the sensitivity of esophageal cancer to chemoradiotherapy.

Studies conducted by OU GF et al. (Ou et al., 2003) have shown that the five-year survival rates of the groups respectively revealing severe, moderate and mild reactions as well as the single surgical group after preoperative radiotherapy are respectively $60.7 \%, 46.4 \%, 21.1 \%$ and 
$38.8 \%$. Such a result indicates that the patients highly sensitive to the radiotherapy present an obviously higher survival rate during the preoperative radiotherapy of esophageal cancer than during the single surgery. The survival rate of patients moderately sensitive to the radiotherapy has been improved through preoperative radiotherapy; while the survival rate of patients resistant to the radiotherapy after preoperative radiotherapy decreases compared with patients in a single surgical group, and their local relapse rate and distant metastasis rate are also higher than those of the groups revealing severe reaction and moderate reaction as well as the single surgical group. Thus, we can draw a conclusion that it is not effective or even harmful for patients non-sensitive to the radiotherapy to receive preoperative radiotherapy. The result of this study showed that the two-year survival rates of the groups revealing severe and mild reactions were respectively $61.9 \%$ and $33.3 \%$. Therefore, among the patients with a declining serum VEGF level, there were respectively $61.1 \%$ patients in the group revealing severe reaction and $33.3 \%$ patients in the group revealing mild reaction showing an overall survival of more than 2 years. Among patients with a rising serum VEGF level, there were respectively $33.3 \%$ patients in the group revealing severe reaction and $66.7 \%$ patients in the group revealing mild reaction showing an overall survival of less than 2 years. It is implied that the patients in the group revealing severe reaction are highly sensitive to chemoradiotherapy; if their serum VEGF level declined they would present a more effective status in long-term survival. While the patients in the group revealing mild reaction were nonsensitive to chemoradiatherapy, and if their serum VEGF level increased, their prognosis would be poor, and they would present a relatively short overall survival. Such a result is consistent with a previously published study $(\mathrm{Ou}$ et al., 2003),which suggested that, the more severe the pathological reaction of postradiotherapy tumor tissue, the better the long-term survival would be and the higher the effective rate in the patients with a declining serum VEGF level after treatment. However, this study is restricted by insufficient samples and abundant censored data, so there has no statistical difference when conducting the intergroup contrastive analysis. The study still needs more samples and an extended follow-up period for further study. As for patients with a rising serum VEGF level, the anti-tumor angiogenesis drugs (Limaverde-Sousa et al., 2014) which target VEGF and its receptors (such as Thalidomide (Yu et al., 2011)) are adaptable to reduce the serum VEGF level and extend their overall survival.

In conclusion, the results of this study indicate that the pathological reaction of the tumor tissue after the chemoradiotherapy was closely related with long-term survival. The change of serum VEGF level during treatment not only can become a useful index to judge the prognosis of esophageal cancer, but also may act as a helpful marker to predict their sensitivity to chemoradiotherapy. With the ongoing course of clinical study, through various comprehensive clinical, imagebased and laboratory data, the sensitivity of esophageal cancer to chemoradiotherapy may be predicated and evaluated based on individualized treatment.

\section{Acknowledgements}

This work was supported by Guiding Science Research Project of The Health Department of Jiangsu Province (Z201220), Major Project of the Health Department of Changzhou (ZD201105), and Sci-Tech Supporting Society Development Project of Changzhou (CE20125021).

\section{References}

Angelescu C, Burada F, Ioana M, et al (2013). VEGF-A and VEGF-B mRNA expression in gastro-oesophageal cancers. Clin Transl Oncol, 15, 313-20.

Brun E, Zatterstrom U, Kjellen E, et al (2001). Prognostic value of histopathological response to radiotherapy and microvessel density in oral squamous cell carcinomas. Acta Oncol, 40, 491-6.

Cellini F, and Valentini V (2014). Targeted therapies in combination with radiotherapy in oesophageal and gastroesophageal carcinoma. Curr Med Chem, 21, 990-1004.

Crehange G, Maingon P, Peignaux K, et al (2007). Phase III trial of protracted compared with split-course chemoradiation for esophageal carcinoma: Federation Francophone de Cancerologie Digestive 9102. J Clin Oncol, 25, 4895-901.

Jiang JT, Zhang LF, Zhou B, et al (2012). Relationships of uPA and VEGF expression in esophageal cancer and microvascular density with tumorous invasion and metastasis. Asian Pac J Cancer Prev, 13, 3379-83.

Limaverde-Sousa G, Sternberg C, and Ferreira CG (2014). Antiangiogenesis beyond VEGF inhibition: a journey from antiangiogenic single-target to broad-spectrum agents. Cancer Treat Rev, 40, 548-57.

Liu J,Huang XE (2014). Efficacy of bifidobacterium tetragenous viable bacteria tablets for cancer patients with functional constipation. Asian Pac J Cancer Prev, 15, 10241-4.

Ou GF, Wang M, Wang LH, et al (2003). [Relation between pathologic tumor response to preoperative radiotherapy and the prognosis in patients with esophageal carcinoma]. Zhonghua Zhong Liu Za Zhi, 25, 278-81.

Peng J, Shao N, Peng H, and Chen LQ (2013). Prognostic significance of vascular endothelial growth factor expression in esophageal carcinoma: a meta-analysis. $J$ BUON, $\mathbf{1 8 ,}$ 398-406.

Qian YD, Xu X, Wang L, et al (2014). Clinical safety of chemotherapy for elderly cancer patients complicated with hypertension. Asian Pac J Cancer Prev, 15, 9875-7.

Shibata Y, Haruki N, Kuwabara Y, et al (2002). Chfr expression is downregulated by $\mathrm{CpG}$ island hypermethylation in esophageal cancer. Carcinogenesis, 23, 1695-9.

Srivastava VK, Gara RK, Rastogi N, et al (2014). Serum vascular endothelial growth factor-A (VEGF-A) as a biomarker in squamous cell carcinoma of head and neck patients undergoing chemoradiotherapy. Asian Pac J Cancer Prev, 15, 3261-5.

Stahl M, Walz MK, Stuschke M, et al (2009). Phase III comparison of preoperative chemotherapy compared with chemoradiotherapy in patients with locally advanced adenocarcinoma of the esophagogastric junction. J Clin Oncol, 27, 851-6.

Tian GY, Miu M, Huang XE (2014).Systematic analysis of pemetrexed-based chemoradiotherapy for patients with locally advanced or metastatic esophageal cancer. Asian Pac J Cancer Prev, 15, 8475-8.

Wang W, Sun QK, He YF, et al (2014). Overexpression of periostin is significantly correlated to the tumor angiogenesis and poor prognosis in patients with esophageal squamous 
Jing-Ping Yu et al

cell carcinoma. Int J Clin Exp Pathol, 7, 593-601.

Xiao Y, Liu J, Huang XE, et al (2014).Clinical study on fluvoxamine combined with oxycodone prolonged-release tablets in treating patients with moderate to severe cancer pain. Asian Pac J Cancer Prev, 15, 10445-9.

Yu J, Liu F, Sun Z, et al (2011). The enhancement of radiosensitivity in human esophageal carcinoma cells by thalidomide and its potential mechanism. Cancer Biother Radiopharm, 26, 219-27. 